\title{
The thermal explosion synthesis of AlNi monitored by neutron thermodiffractometry
}

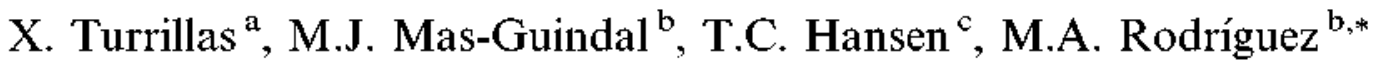 \\ "CISDEM, CSYC, CI Serrano Galvache 4, 28033 Madrid, Spain \\ ${ }^{b}$ Instituto de Cerámica y Vidrio, CSIC, Ct Kelsen 5, 28049 Madrid, Spain \\ ${ }^{c}$ Institut Laue-Langevin BP 156, 6, ne Jules Horowitz, F-38042 Grenoble Cedex 9. France
}

\begin{abstract}
The synthesis of AlNi from thermally activated equimolar powder mixtures of aluminium and nickel was monitored in situ and acquired diffraction patterns every $2 \mathrm{~s}$ or less. The analysis of diffraction patterns $(\lambda=1.3112 \AA)$ permitted establishment of its kinetics, which could be modeled according to an equation expressed as $k t=[\alpha /(1-\alpha)]^{3 / 2}$. From 1530 to $\approx 600$ " $\mathrm{C}$ an activation energy of $9 \pm 2 \mathrm{~kJ} \mathrm{~mol}^{-1}$ was estimated. Also, the crystallite size evolution on cooling was established to vary from $\approx 14 \mathrm{~nm}$ at $1530^{\circ} \mathrm{C}$ to $\approx 28 \mathrm{~nm}$ at $180^{\circ} \mathrm{C}$. Finally, the reaction was found to occur through the melting of aluminium and the subsequent dissolving of nickel to form the polycrystalline single-phase product, AlNi.
\end{abstract}

Kevwords: Nickel aluminides; Neutron diffaction: Nucleation and growth; Thermodiffraction

\section{Introduction}

Nickel aluminides appear to have very good properties that make them eligible for the development of new materials. They have excellent oxidation resistance, thermal stability and strength at high temperature. The most promising phase is $\mathrm{AlNi}$; it has a melting point of $1638{ }^{\circ} \mathrm{C}$, relatively low density $\left(5910 \mathrm{~kg} \mathrm{~m}^{-3}\right)$, excellent corrosion resistance and good thermal conductivity $\left(75 \mathrm{~W} \mathrm{~m}^{-1} \mathrm{~K}^{-1}\right)$. These properties make it useful for a wide range of engineering applications that involve coating of materials such as surface catalysts, semiconductors or other materials to make them corrosion-resistant. They are also widely employed for structural applications such as turbine blades and vanes in the automotive or aeronautical industry, where lightweight components are in high demand $[1,2]$.

Concerning their production, the main routes used for other intermetallics, such as casting, rapid solidification,

\footnotetext{
Corresponding author.

E-mail address: mar@icv.csic.es (M.A. Rodríguez).
}

mechanical alloying and powder processing, are also applicable to nickel aluminides. However, there is an interesting route that has been recently explored with very good results: Self-propagating High-temperature Synthesis (SHS). The recycling of the energy released from highly exothermic reactions provides the advantage of sustaining a process as a combustion or reaction wave. The main characteristics of this process are its low processing cost and the energy- and time efficiency savings, and these have made SHS a very attractive route for the production of a very wide range of materials. Examples of industrial compounds produced by this method are: refractories (carbides, nitrides, silicides, borides), complex oxides, hydrides, chalcogenides andintermetallics [3-11].

Usually, the reaction is initiated by a triggering heat source, which is in contact with the reactive powder mixture. The energy released from the exothermic reaction makes selfpropagation possible through the whole sample [10]. When the process is not very favoured, there are several ways to activate the process, like mechanical alloying SHS, which is based in the activation of the reactant mixture through 
energetic milling $[10,12,13]$, or chemical activation SHS, based on the use of a secondary reaction to supply the necessary energy for the self-sustaining process $[10,14]$.

Regarding the production of nickel aluminides, SHS has received considerable attention because the reactions are highly exothermic and very favoured, it thus being possible to produce them with relative ease. At this point, it is necessary to point out the differences between SHS and thermal explosion routes. In SHS mode, the reaction starts with the help of a small burst of energy and propagates as a wave front from one initial point to the rest of the reactive mixture. The temperature gradients created as the wave advances are very steep, the cooling is faster than in thermal explosion mode, the diffusion processes are slower and the system is very far from equilibrium. Consequently, the product contains a number of intermediates, and the crystallites are smaller. In contrast, in the case of a thermal explosion synthesis, the whole reactive mixture is heated and the reaction takes eventually place as a sudden mild explosion. This way, although still not in equilibrium, is closer to it than is the case with SHS. The final product cools down more slowly and is more homogeneous, and the crystallites are larger. The energy consumption is quite different as well - it is higher in thermal explosion mode. Finally, the ancillary for the SHS is simpler; only a closed recipient to control the gases surrounding the reactants suffices, while for thermal explosion a specially designed furnace is necessary.

Considerable efforts have been devoted to understanding the mechanism of SHS and to determine the different stages occurring during these reactions. The main difficulties in achieving such understanding are related to the high rate of reactions, the high temperatures at which reactions take place and the lack of direct experimental methods with which to study them [15]. A common attempt to overcome these limitations consists of stopping the reaction by quenching and then examining the existing phases at each stage $[16,17]$. However, this approach has a severe limitation since it is very difficult to control the time. A more effective approach is to use in situ measurement techniques, which permit the phases involved in the reaction to be monitoring as they form or react, without external interference. Two of the most widely used techniques are X-ray and neutron diffraction. Time-resolved X-ray diffraction has been used mainly with high-energy synchrotron radiation due to its superior resolution [8]. It has been used mainly on thin samples or on the surface of bulk samples $[18,19]$. Neutron radiation has the advantage of having superior penetration, so diffraction data can be acquired from thicker specimens. Although, in the past, longer acquisition times were necessary to get diffraction data comparable to the ones obtained by synchrotron X-ray, today some neutron instruments can provide diffraction patterns with $1 \mu$ s time resolution. In situ time-resolved neutron diffraction (TRND) has been used, for instance, to study the mechanism of synthesis of titanium silicon carbides [20-23].

The nickel-aluminium combustion synthesis mechanism was studied by Varma et al. [24] using in situ diffraction techniques. They concluded that the reaction was triggered by the melting of aluminium, then an intermediate phase appeared and finally the product formed at approximately $20 \mathrm{~s}$ after the passage of the front. Evidence of the presence of intermediates during the reaction was confirmed by Curfs et al. [25], also by using synchrotron radiation, but AlNi was the first phase to appear, followed by the secondary phases $\mathrm{AlNi}_{3}, \mathrm{Al}_{3} \mathrm{Ni}_{2}$ and $\mathrm{Al}_{3} \mathrm{Ni}_{5}$. Despite the importance of this system, as explained earlier, no experiments studying the reaction mechanism using TRND have been reported. The objective of this paper is to report in situ neutron diffraction studies of the thermally activated combustion synthesis of AlNi from its elements.

\section{Experimental}

\subsection{Starting materials and sample preparation}

The following starting materials were used: (i) carbonyl nickel-powder Type 123>99\% (William Rowland Ltd., UK), with particle size $d_{50}=5 \mu \mathrm{m}$ and specific surface $S_{s}=0.4 \mathrm{~m}^{2} \mathrm{~g}^{-1}$ and (ii) Al powder $>99 \%$ (Alcoa, USA), with particle size $d_{50}=55 \mu \mathrm{m}, S_{s}=0.72 \mathrm{~m}^{2} \mathrm{~g}^{-1}$.

The products were weighed out, mixed thoroughly according to a $1: 1$ stoichiometry in a porcelain mortar and axially pressed in a $15 \mathrm{~mm}$ diameter stainless steel die. A pressure of $20 \mathrm{MPa}$ was applied to obtain $15 \mathrm{~mm}$ thickness cylindrical pellets of approximately $6 \mathrm{~g}$.

\subsection{Time-resolved neutron diffraction experiments}

Neutron diffraction patterns were acquired at the instrument D20 of the Institut Max von Laue-Paul Langevin (Grenoble, France), operating a High Flux Reactor at $58 \mathrm{MW}$ thermal power. D20 is a high-flux medium-resolution powder diffractometer of 1536 cells that allows fast data acquisition up to a $2 \Theta$ of $155^{\circ}$ [27]. A neutron wavelength of $1.3112 \AA$ was selected, using a copper (200) monochromator at a take-off angle of $42^{\circ}$. The precise wavelength was determined by refining the diffraction pattern of a NBS Si standard. The neutron beam was collimated for a rectangular section of $40 \times 14 \mathrm{~mm}$.

Pressed powders were piled up in the centre of a silica tube of $16 \mathrm{~mm}$ of inner diameter with a $1.5 \mathrm{~mm}$ thick wall. The tube was placed inside a niobium cylindric furnace and insulated from the base, as described elsewhere $[22,26]$.

Specimens were set under high-vacuum and heated up at linear rates ranging from 10 to $15^{\circ} \mathrm{C} \mathrm{min}{ }^{-1}$ until the reaction started and propagated, while recording a diffraction pattern every $421 \mathrm{~ms}$ in some experiments and every $2.21 \mathrm{~s}$ in others to get better diffraction statistics but with poorer time resolution. Once the reaction was initiated, the heating element was turned off and the system was allowed to cool while diffraction patterns were acquired at same pace as before. Before heating and after cooling, diffraction patterns were recorded for 2 min to help identify reactants and products. 


\subsection{Characterization by scanning electron microscopy ( SEM)}

Products were crushed and embedded in epoxy resin under vacuum. Polishing was done on a lapping disk with silicon carbide paper down to P2500 size, then with diamond powder down to $3 \mu \mathrm{m}$ size. The polished surface was then cleaned with isopropyl alcohol, dried and coated by gold sputtering for examination by field emission SEM (HITACHI S-4700 microscope, Japan).

\subsection{Data processing}

Visual analysis of the phase transformations and the reaction mechanism was performed using the large-array manipulation program Transform [28]. Data conditioning and global fit to Gaussian were carried out with the help of code written in IDL [29]. General plotting and data fitting was done with Origin 8.0 [30]. Rietveld refinements were accomplished with Fullprof [31] and the Reflex module of Materials Studio 4.3 [32]. With this latter package, Pawley refinements were also done. Thermodynamic calculations were made with the package HSC [33] by the Gibbs energy minimization method. Various enhancements, corrections, tracing and quantifications on SEM photographs were done with the help of Image Pro [34].

\section{Results and discussion}

\subsection{Thermodynamic predictions}

The Al-Ni phase diagram [35] (Fig. 1) shows five stoichiometric species: $\mathrm{Al}_{3} \mathrm{Ni}, \mathrm{Al}_{3} \mathrm{Ni}_{2}, \mathrm{Al}_{3} \mathrm{Ni}_{5}, \mathrm{AlNi}_{3}$ and $\mathrm{AlNi}$.

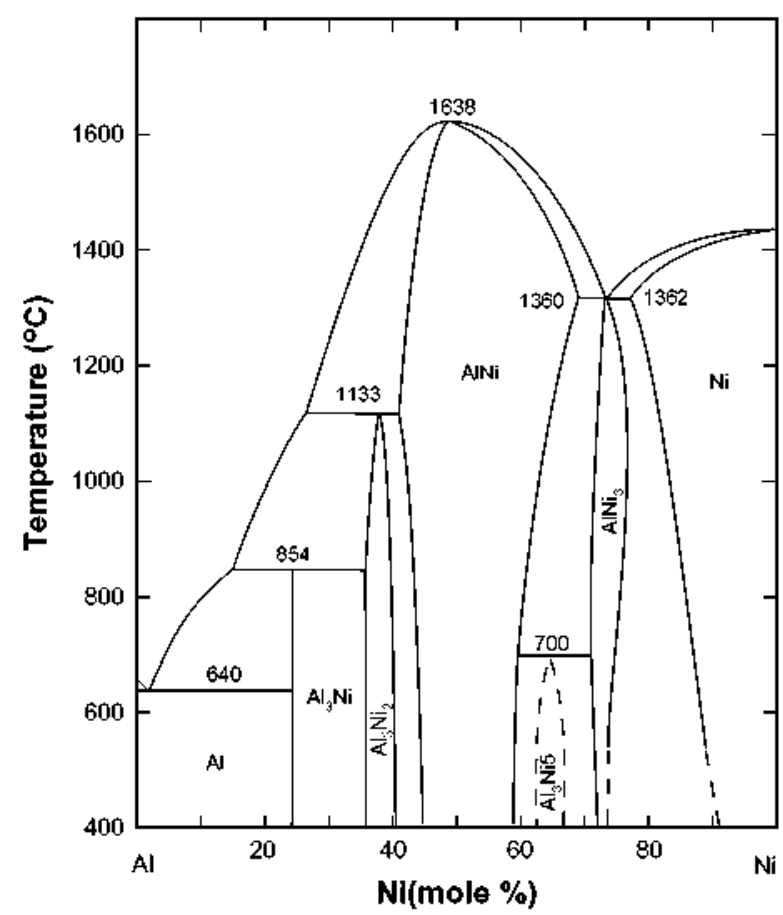

Fig. 1. The phase diagran of Al-Ni. It is an adaptation of the original published by Nash et al. [35].

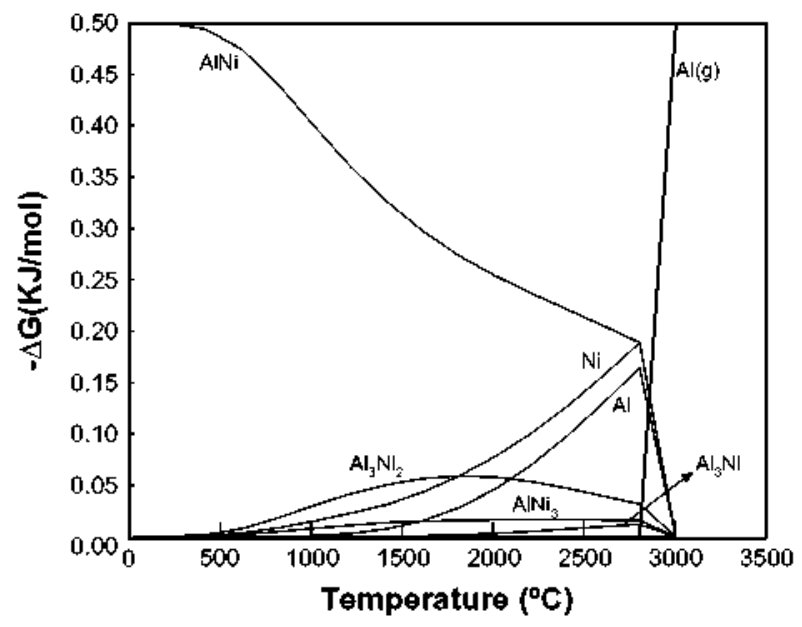

Fig. 2. Diagram of the thermodynanic relationships between the different phases existing within the Al-Ni system. calculated with the help of HSC [33].

AlNi melts congruently and is the most favoured species to be formed. This was confirmed in two ways. First, thermodynamic calculations made with HSC software (Fig. 2), based on Gibbs energy function calculations, indicated that $\mathrm{AlNi}$ is the most favoured phase, followed by $\mathrm{Al}_{3} \mathrm{Ni}$ and the metals at a higher temperature. Secondly, when another thermodynamical criterion is used to evaluate whether or not a combustion reaction is favoured, such as the adiabatic temperature (the temperature that a system would reach if it had no energy exchange with the exterior), the same conclusion is reached.

The results published by Curfs et al. [25] confirmed these calculations and the presence of $\mathrm{Al}_{3} \mathrm{Ni}_{5}$ in the diffraction patterns of final products. Both $\mathrm{Al}_{3} \mathrm{Ni}_{2}$ and $\mathrm{Al}_{3} \mathrm{Ni}_{5}$ are next to AlNi formation zone, which explains why they appear so easily.

Other authors empirically established that the reaction would be self-sustaining if the adiabatic temperature was higher than $1800 \mathrm{~K}$ [36]. According to those data, the adiabatic temperature for AlNi is $1910 \mathrm{~K}$ [24], while for the other species it is lower than $1600 \mathrm{~K}$.

\subsection{In situ neutron diffiaction experiments}

One of the experiments is shown in Fig. 3. The graph consists of a sequence of 6682 diffraction patterns. Each one is acquired after $0.2 \mathrm{~s}$ of counting with a dead time of $0.22 \mathrm{~s}$. They are displayed as a three-dimensional map (on top) and its projection (on bottom) as a contour map in two dimensions. This is a convenient way of showing major changes such as reactions or phase transitions, or, as in this case, peak shifts due to thermal expansion. The mixture of $\mathrm{Al}$ and $\mathrm{Ni}$ was heated at a rate of $15^{\circ} \mathrm{C} \mathrm{min}^{-1}$, and when a temperature of $660 \pm 20^{\circ} \mathrm{C}$ was reached the reaction suddenly took place. This temperature was measured from the thermal expansion of nickel present in the mixture and coincides with the $\mathrm{Al}$ melting point. It is important to 

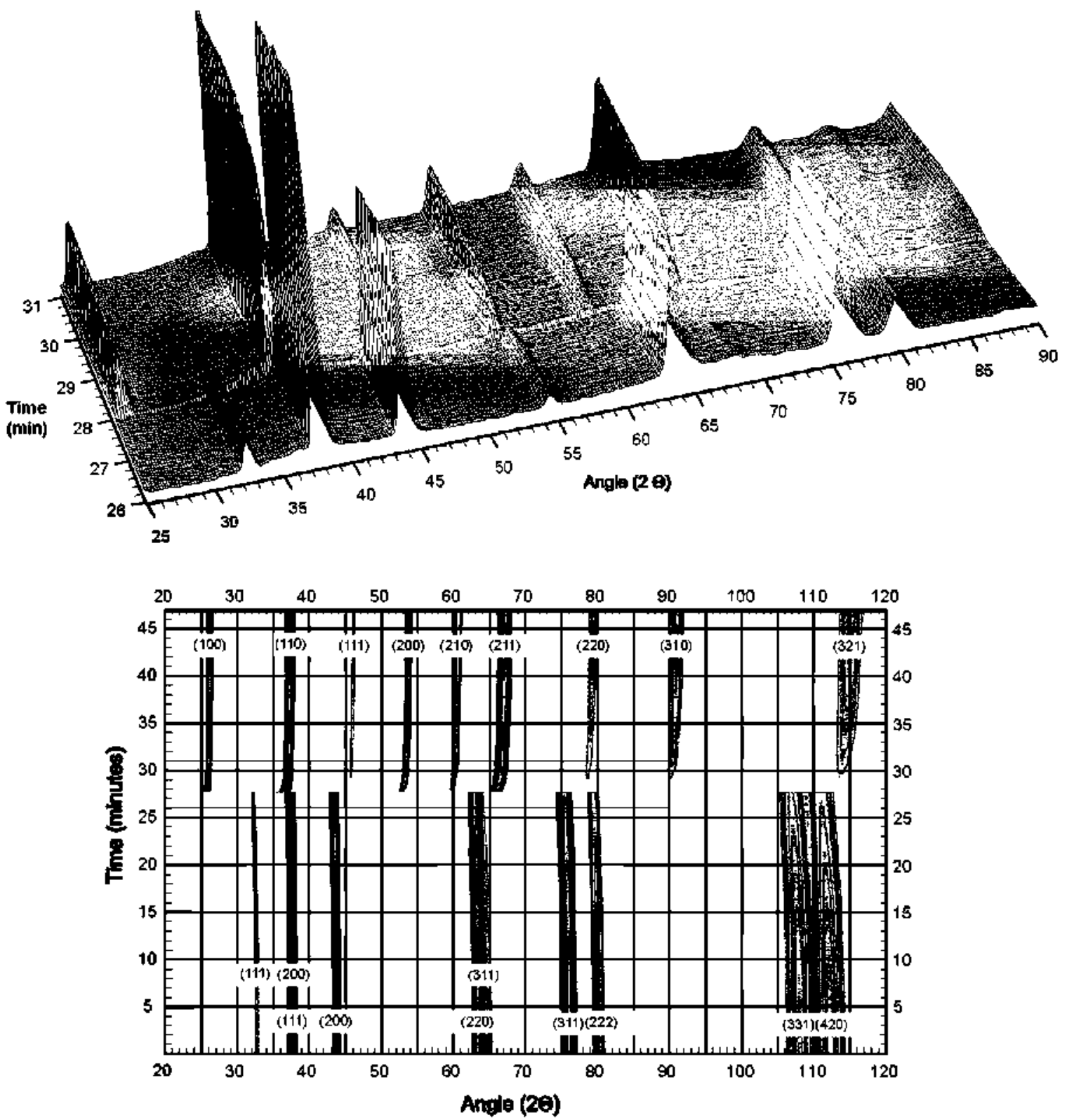

Fig. 3. The sequence of the reaction of $\mathrm{Al}$ and $\mathrm{Ni}$ to form $\mathrm{AINi}$. Top: detail corresponding to the area (highlighted on the bottom) where the reaction takes place. The inferior part of the contour map corresponds to the three-dimensional representation of the diffraction patterns series. The Miller indices for each phase are superimposed; nickel at the bottom. aluminium in the middle and AlNi at the top.

mention that in all the cases in which this combustion was monitored - regardless of the heating ramp followed - the ignition started at temperatures lower than $750^{\circ} \mathrm{C}$.

\subsubsection{Identification of phases}

Only $\mathrm{Al}, \mathrm{Ni}$ and AlNi were detected. A Rietveld refinement of the diffraction pattern (acquired for 2 min) was performed at the end of the experiment, when the temperature was stable. Only AlNi was present. Fig. 4 shows the results. The peak shape was fitted to a Thompson-CoxHastings curve. Asymmetry was fitted to a Bérar-Baldinozzi model and the background to a 20 degree polynomial.

\subsubsection{Temperature profiles obtained from diffraction data}

The $(110),(211),(220)$ and ( 310$)$ reflections were fitted to simple Gaussian peak shapes for all diffraction patterns acquired on cooling using code written in IDL, as explained before. The same statistical weighting was attributed to them all. Although they appear at relatively low angles and therefore their intrinsic error - linked to the nature of the Bragg law - is large, their intensity is quite strong. Their positional accuracy is counterbalanced by their better statistics for Gaussian fitting. In any case, the uncertainty associated is lower than that required to estimate the temperatures within an error of $\pm 10^{\circ} \mathrm{C}$. The calculations are based on the thermal expansion data quoted 


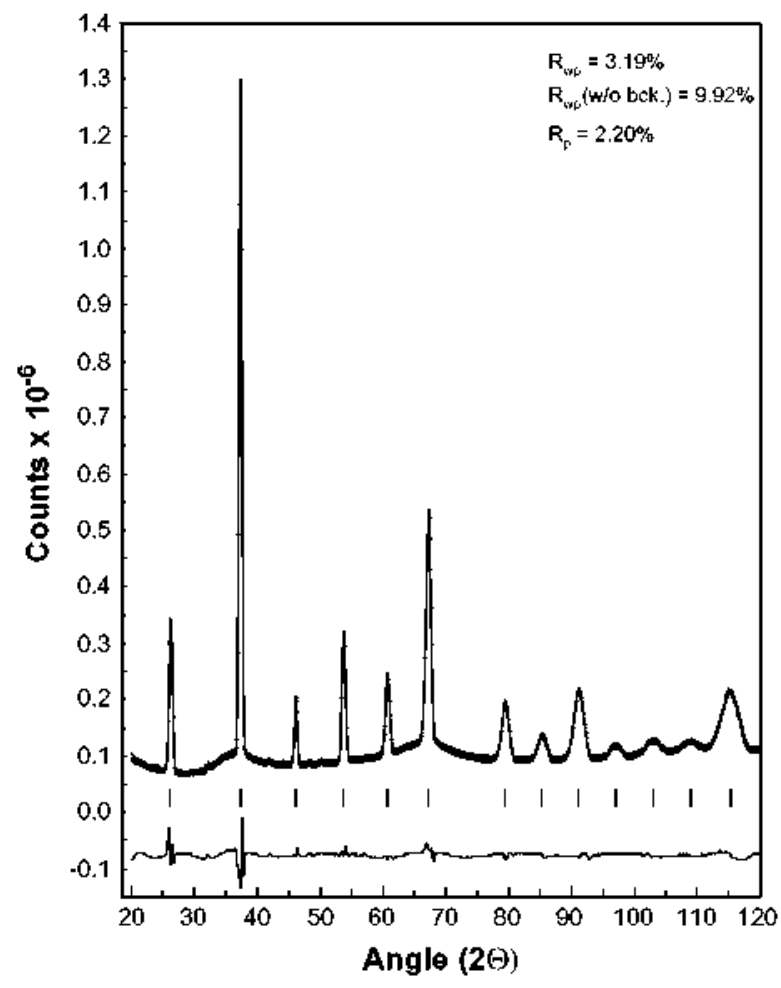

Fig. 4. Rietveld refinement plot of AlNi at the end of the experiment, displayed in a conventional way. The statistical indicators can be seen at the top right.

in Ref. [37]. The final temperature at the end of the experiment was measured with a thermocouple $\left(180^{\circ} \mathrm{C}\right)$. From this value the temperatures were scaled up to obtain a maximum temperature of $1530^{\circ} \mathrm{C}$ for the first diffraction pattern detected after ignition. The curve profile does not show any deviation from a model that takes into account both radiation and convection effects. The experimental values can be seen along with the fitted data in Fig. 5. The actual formula used was

$i=\frac{k_{4}-T}{k_{1} T^{4}+k_{2} T+k_{3}}$

where $k_{1}, k_{2}, k_{3}$ and $k_{4}$ are constants to be fitted and $t$ stands for time and $T$ for absolute temperature.

The derivation of this equation can be found in Ref. [8], where a similar approach was used to fit $\mathrm{T}_{1} \mathrm{~B}_{2}$ synchrotron diffraction data while cooling.

\subsubsection{Estimation of activation energy for the crystal growth of $\mathrm{AlNi}$}

It is quite clear that there is a progressive growth of reflections of $\mathrm{AINi}$, leading to the complete reaction of $\mathrm{Al}$ and $\mathrm{Ni}$, as shown in Fig. 3. It is also possible to quantify this growth by measuring the intensities of the reflections. For this particular task, the (110) reflection was selected since it is a single, well-resolved and reasonably intense reflection (the strongest) to be fitted to a Gaussian shape. The variation in intensity for this reflection as a function of time is related to the crystal growth of AlNi on cooling.

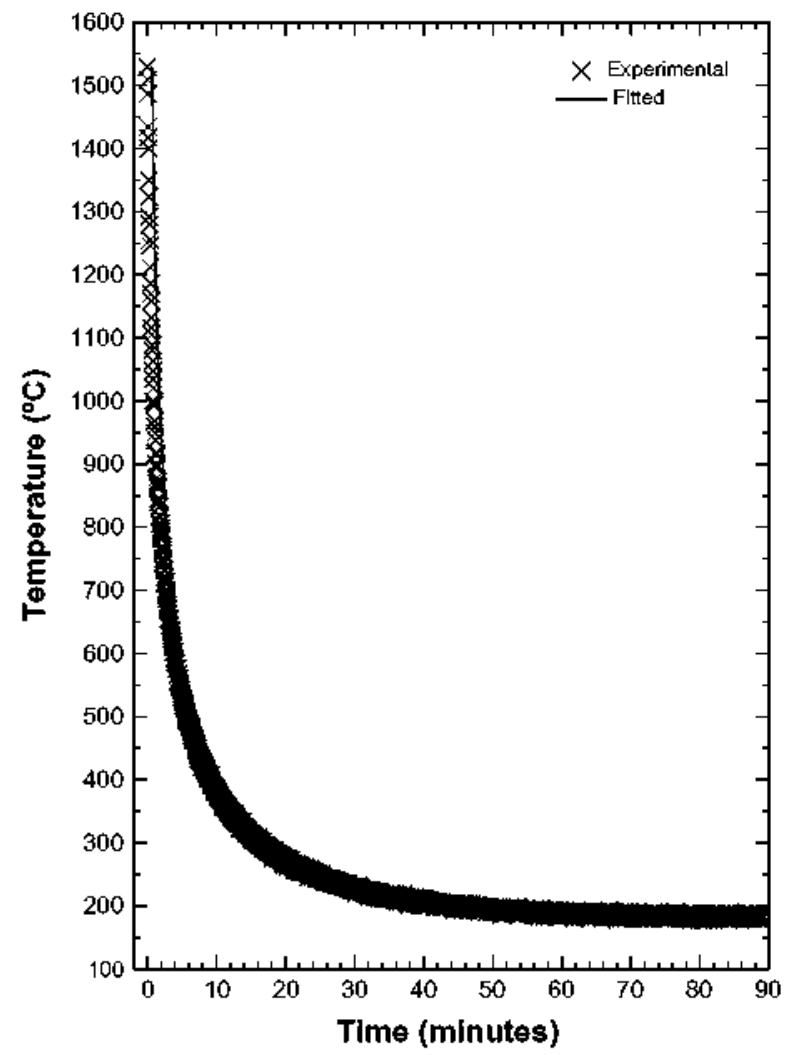

Fig. 5. The evolution of temperature from the moment that the reaction took place. The temperature falls in a regular way and can be fitted (continuous line) to a theoretical model explained in the text.

It can be seen in a normalized way (scaled to 1 at the end of the reaction) in the right-hand projection plane of Fig. 6 . This approach to monitoring the phase formation by monitoring the intensity variation of a single reflection is well documented. More details about the underlying theory can be found in the book of Chung et al. [38] dedicated to high temperature diffraction analysis. More recently an illustrative treatment of neutron diffraction data in order

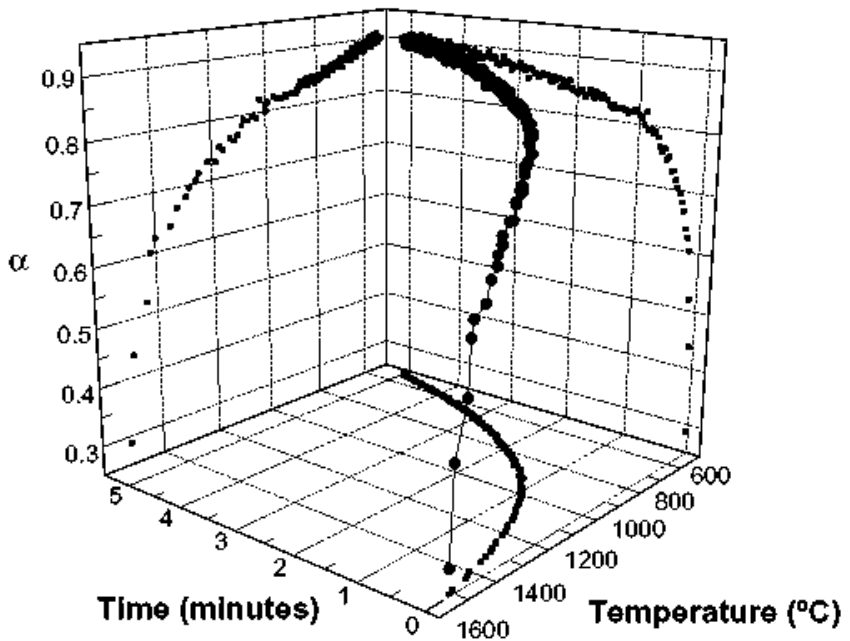

Fig. 6. Three-dimensional representation of three variables involved in the experiment. The progress of the reaction $(\alpha)$. the time and the temperature. The projections on the planes represent their dependence by pairs. 
to establish the kinetics of a reaction can be found in the work of Riley et al. [23].

In order to study the kinetics in a solid-state reaction, it is customary to represent the progress of a reaction by a dimensionless parameter alpha, going from 0 to 1 , when the reaction is completed. According to this convention, a generic kinetics model is expressed by the equation

$k i=f(\alpha)$

Different $f(\alpha)$ models have been described for solid-state reactions. A quite exhaustive list can be found in the book edited by Bamford et al. [39]. The usual approach to finding the most likely model, when it is totally unknown, is to try to plot and fit the experimental values for different possible models. This is valid for reactions taking place at constant temperature. Sometimes this is not possible, or the temperature is deliberately varied in a controlled way, usually keeping a linear rate $(\Delta T / \Delta t=$ constant $)$ to obtain activation energies in a single experiment.

In this particular case, the temperature decreases in a monotonic way, and fortunately the temperature is known along the whole experiment, since the cell parameters of AlNi can be measured with adequate precision. Bearing in mind that the reaction constant follows an Arrhenius behaviour, the previous Eq. (2) can be rewritten as

$A \exp \left(\frac{-E_{0}}{R T(t)}\right) t=f(\alpha)$

where $A$ represents the pre-exponential factor also known as the frequency factor, $E_{a}$ is the activation energy and $R$ is the universal gas constant $\left(8.31434 \mathrm{~J} \mathrm{~mol}^{-1} \mathrm{~K}^{-1}\right)$.

Basically there are three variables to relate: time, temperature and $\alpha$. Finding an equation that relates them all would allow us to determine the activation energy. A three-dimensional graph of these three variables can be seen in Fig. 6, where projections on the three planes show the corresponding two-dimensional dependencies by pairs of variables.

As explained before, different hypothetical models were tested - including the popular Avrami-Erofe'ev model but most of them either did not fit well or gave meaningless values such as negative activation energies. Therefore they were discarded and in the end a simple expression was used:

$k t=\left(\frac{\alpha}{1-\alpha}\right)^{n}$

The practical way to fit the data was to take logarithms in Eq. (4) and to include Eq. (3) to obtain the expression,

$\ln \frac{\alpha}{1-\alpha}=\frac{\ln A}{n}-\frac{E_{0}}{1000 R n} \frac{1000}{T}+\frac{\ln t}{n}$

If the variables $x, y$ and $z$ are defined as $x \equiv 1000 / T, y \equiv \ln t$ and $z \equiv \ln (\alpha /(1-\alpha))$, we can obtain a simple equation of a plane in three dimensions:

$z=z_{o}+a x+b y$

where $z_{o}=\ln A / n, a=E_{a} / 1000 / R / n$ and $b=1 / n$.

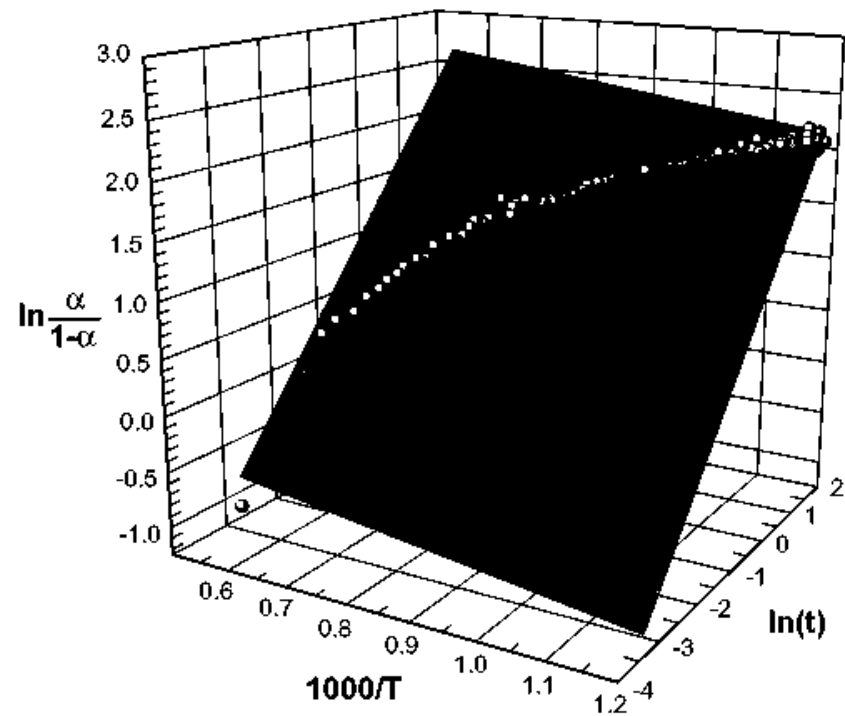

Fig. 7. Three-dimensional representation of the variables involved in the calculations of the kinetics model. The values represented as spheres are fitted to the plane represented as a grid.

The experimental tabulated points $(x, y, z)$ can be plotted in three dimensions and a plane fitted to them. This is shown in Fig. 7. The regression coefficient was $R^{2}=0.98$ and the relevant parameters deduced as $A=17.6 \pm 2 \mathrm{~min}^{-1}, E_{\mathrm{o}}=$ $9 \pm 2 \mathrm{~kJ} \mathrm{~mol}^{-1}$ and $n \simeq 3 / 2 \pm 0.2$. Therefore, the kinetic model could be formulated in an approximate way by the equation

$k t=\left(\frac{\alpha}{1-\alpha}\right)^{3 / 2}$

The apparent activation energy for the crystal growth is defined for a wide temperature interval (from 1530 to $628^{\circ} \mathrm{C}$ ) for a conversion $(\alpha)$ from 0.29 to 0.90 . This value has to be taken with caution since it is based upon a number of hypotheses, as mentioned before (Arrhenius behaviour and the validity of the model in a wide temperature range). It only gives an idea of how favoured the formation of the AlNi crystalline phase is. Its low value indicates that the reaction is indeed very favoured.

\subsubsection{Estimation of crystallite size on cooling}

It is well known that powder diffraction patterns contain information about crystallite size. Basically, the breadth of the diffraction peaks are related to the size of the crystal domains by the Scherrer equation,

$\beta_{h k l}=\frac{K \lambda}{L \cos \theta_{h k !}}$

where $\beta_{h H}$ is the full width at half maximum (FWHM) of the diffraction peak in radians - due to the particle size broadening contribution to the peak width $-K$ is a constant that varies with the method of taking the breadth $(0.89<K<1), \lambda$ is the wavelength of incident radiation, $\theta_{h k J}$ is the angle of the peak and $L$ is the crystallite length. Modern computing crystallography packages use this 


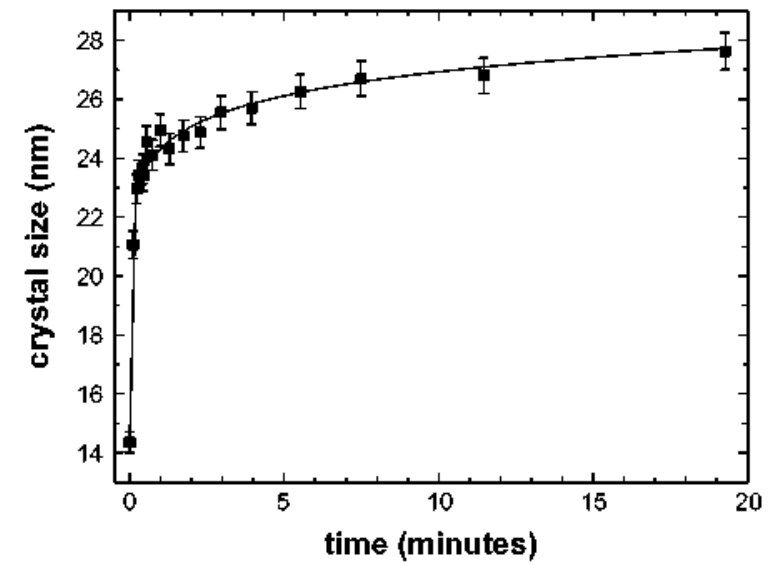

Fig. 8. The AlNi crystallite size variation as a function of time while cooling. determined from the peaks' breadths. The continuous line represents the fitted curve.

equation to estimate crystalline sizes using all the diffraction peaks present in a diffraction pattern.

Unfortunately, in most cases the values obtained this way are subjected to large systematic errors; they are therefore useful in seeing the relative variations in a series of diffraction patterns collected under the same conditions. In the present case, a Pawley refinement with the Reflex module of Materials Studio [32] was performed. First of all, from the silicon standard diffraction pattern, the instrumental broadening was determined assuming that the crystallite size of the silicon powder was sufficiently large to ignore its contribution to the increase in FWHM.

Diffraction patterns at regular intervals of temperature were refined according to the Pawley scheme. Instrumental parameters obtained from a silicon standard refinement were kept fixed, while the cell parameters and crystalline broadening were refined. For the peak profile a Thompson-Cox-Hastings function was used. The results are plotted in Fig. 8, where crystallite size, expressed in $\mathrm{nm}$, is represented as a function of time. The continuous line represents the fitted curve. The square root of time dependence was tested first - consistent with a diffusion process - but it did not fit very well. Therefore, a more generic equation of the type $l=k t^{n}$ was used. The data seem to fit reasonably well $\left(R^{2}=0.988\right)$ when $k=24.3 \pm 0.2$ and $n=0.044$ \pm 0.006 . It could be argued that the physical meaning of this equation is questionable. This odd behaviour is probably the consequence of a complex growth, coupled to the fact that two variables - time and temperature - were not independently controlled.

As expected, $70 \%$ of the total growth takes place over the first $200^{\circ} \mathrm{C}$, then the size increase slows down, behaving almost linearly.

\subsection{Morphological studies}

The images obtained by SEM are shown in Fig. 9. The general image (Fig. 9a) shows an apparently relatively homogeneous surface. Upon magnification and contrast
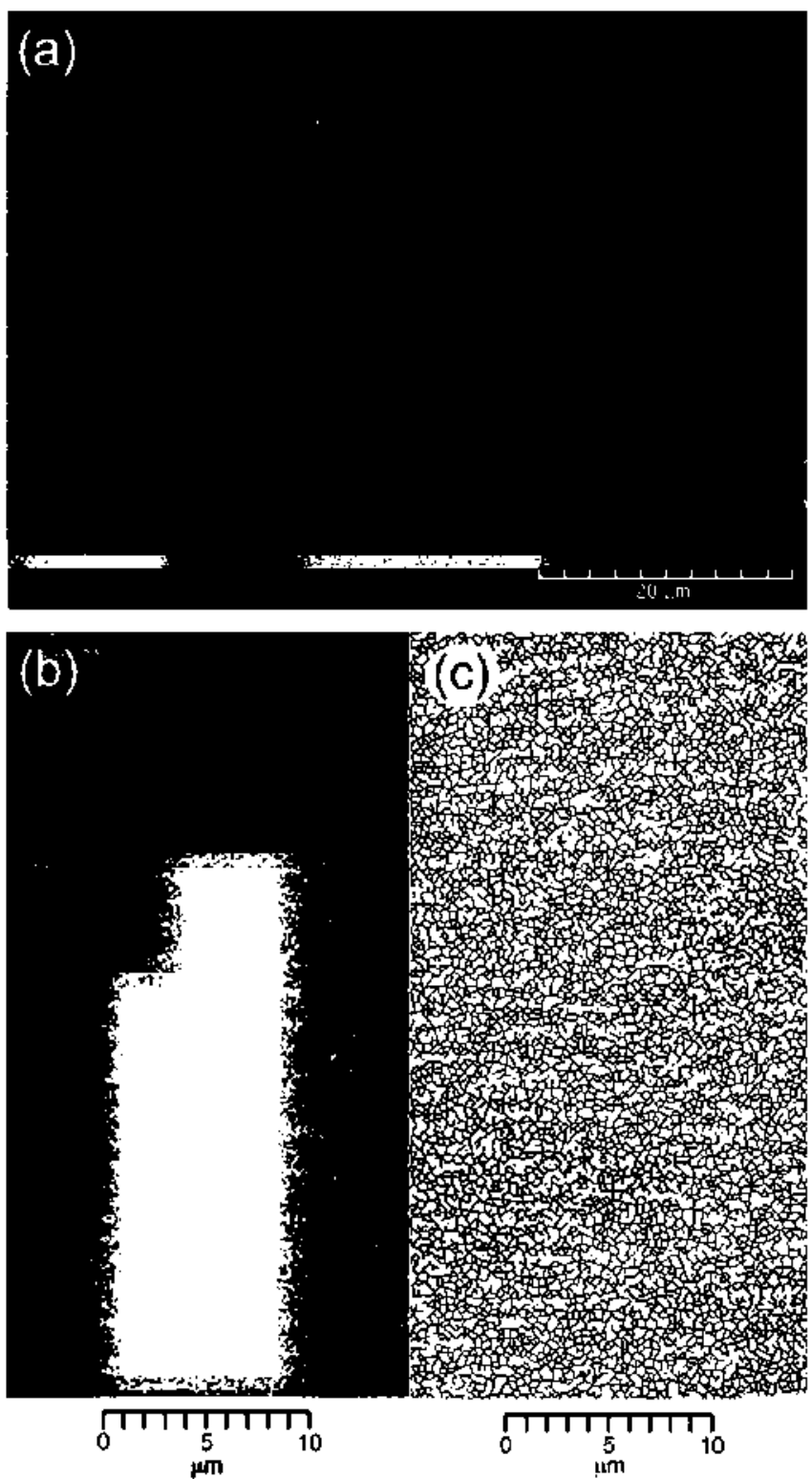

Fig. 9. SEM micrograph of AINi obtained after thermal explosion. (a) A general image. with the scale at the bottom. (b) A magnified and enhanced inage to show microstructural details. (c) The previous image traced to view contours of grains.

enhancement followed by a Bell equalization, a more intricate surface full of grains can be seen (Fig. 9b). Further tracing the contours of the image between grains produces the sketch seen in Fig. 9c. This sketch permits quantification of the grain sizes. They are of sub-micron order (see the scale at the bottom). In some cases they are as small as $100 \mathrm{~nm}$. These observations are not in disagreement with the crystallite sizes estimated above (Section 3.2.4), assuming that the grains contain twisted domains or are composed of a few crystallites, as happens in phases with low symmetry. Otherwise, the discrepancy in crystal size could be attributed to a systematic error of the method used. In any case, it should be stressed that the relevant feature is the evolution of crystal size with time - an aspect that 
can only be revealed with the aid of an in situ probing method such as neutron thermodiffractometry.

\subsection{Mechanisms of reaction}

The experimental data gathered from experiments carried out both in the laboratory and in D20 allow an outline of the reaction to be established. When the Al melting temperature is reached some nickel starts to react with the melted aluminium to form the AlNi phase, the most thermodynamically favoured phase. This exothermic reaction provokes the increase in temperature and the melting of the nickel, and also of the AlNi. This is the only way to explain the sudden increase in temperature in the sample, up to at least $1638^{\circ} \mathrm{C}$. After the reaction has taken place, the AlNi phase precipitates on cooling. This sequence can be considered as the mechanism under equilibrium conditions. When some authors described the mechanism $[24,25]$ for normal SHS - without activation - they considered a non-equilibrium situation since the reaction front was advancing and losing energy while at the same time preheating the rest of the sample. For this reason the reaction takes place between a liquid and a solid phase (nickel). This is why other "intermediate" phases are also present. Their existence is due to the lack of homogeneity of the sample, since, when cooled down very quickly, the diffusion processes are slower and the system cannot reach the state foreseen by the phase equilibrium diagram. Furthermore, since the reaction front is smaller than the X-ray beam, the diffraction patterns contain information from the reacting area as well as from areas before and after the reaction. In conclusion, the mechanisms described for those experiments under non-equilibrium conditions, such as SHS, are valid only for propagating mode syntheses.

The mechanism for the thermal explosion can be described by the steps:

(1) Al melting.

(2) $\mathrm{Al}$ (liq) $+\mathrm{Ni} \rightarrow \mathrm{AlNi}+\mathrm{Q}$.

(3) AlNi and molten $\mathrm{Ni}$ (Homogeneous liquid).

(4) Precipitation of AINi and any secondary phases.

\section{Conclusions}

The synthesis of AINi by thermal explosion was studied with the help of a large-scale facility. The in situ diffraction data, which provided statistically representative bulk information, permitted the measurement of reliable temperatures, within time scale limitations, of the whole process. Also, the diffraction intensity provided a means to establish a kinetics model and give a value for the activation energy of the formation of erystalline $\mathrm{AlNi}$ on cooling from 1530 to $600^{\circ} \mathrm{C}$. Furthermore, the peak diffraction breadths allowed the estimation of the crystallite sizes of AlNi and its evolution on cooling. The reaction starts with the melting of aluminium, then some nickel reacts with the melted aluminium to form the AlNi phase. This exothermic reac- tion causes a temperature rise and the melting of nickel and any AlNi already formed. After reaction, the AlNi precipitates on cooling. As explained in Section 1, the mechanism of this synthesis follows a path closer to equilibrium conditions, there are no large temperature gradients and the cooling is slower. In addition, it is independent of the raw material's particle size, compactness and other parameters. Consequently, at the end of the reaction the product consists of a single-phase. For this reason, it can be concluded that this thermal explosion synthesis method is suitable for the preparation of a homogeneous AINi product.

Finally, from an instrumental point of view, it should be mentioned that the characteristics of the neutron source coupled to the acquisition system implemented in D20 allowed us to get from a few thousand diffraction histograms of a single run: (i) the bulk temperature data from the specimen (based on thermal expansion); (ii) quantification of the reaction's progress; (iii) the proposal of a kinetic model that permitted the activation energy to be estimate; and (iv) an estimation of the crystallite growth on cooling.

\section{Acknowledgements}

Beam-time for neutron diffraction experiments was allocated thanks to an ILL Grant (No. 5-25-134). This work was financed by the Spanish Science and Technology Agency (CICYT) under Project No. MAT2004-04923C02-01) and by the European Commission Grant (NAMAMET STREP NMP3-CT-2004-001470). Thanks are also due to Mr. Jacques Torregrossa for the help and assistance in the setup of the ancillary of Instrument D20.

\section{References}

[1] Ohtsu N. Oku M, Obara K. Ito S, Shisido T, Wagatsuma K. Surf. Interface Anal. 2007;39:528.

[2] Plazanet L, Nardou F. J, Mater. Sci. 1998:33:2129.

[3] Merzhanov AG. Ceran Int. 2006:21:371.

[4] Anuradha TV, Ranganathan S. Mimani T, Patil KC. Scr. Mater. 2001:44:2237.

[5] Merzhanov AG. Ceran. Int. 1995;21:371.

[6] Munir ZA, Anselni-Tamburini U, Mater. Sci. Rep, 1989:3:277.

[7] Curfs C, Cano IG, Vaughan GBM, Turrillas X, Kvick A, Rodríguez MA. J. Eur. Ceram. Soc. 2002;22:1039.

[8] Contreras L, Turrillas X, Vaughan GBM, Kvick А. Acta Mater. 2004:52:4783.

[9] Cano IG, Borovinskaya IP. Rodrignez MA. Grachev VV. J. Am. Ceram. Soc. 2002;85:2209.

[10] Mas-Guindal MJ, Contreras L. Turrillas X, Vaughan GBM. Kvick Å, Rodríguez MA. J. Alloys Compd. 2006;419:227.

[11] Moya JS, Iglesias JE, Limpo FJ, Escrina JA. Makhonin NS. Rodríguez MA. Acta Mater. 1997:45:3089.

[12] Uenishi K. Matsubara T, Kambara M. Kobayashi KF. Scr. Mater. 2001:44:2093.

[13] Suryanarayana C. Ivanov E. Boldyrev VV. Mater. Sci. Eng., A 2001;304-306:151.

[14] Licheri R, Orru R, Cao GC. Mater. Sci. Eng., A 2004:367:185.

[15] Zhu P, Li JCM. Liu CT. Mater. Sci. Eng.. A 2002;329:57.

[16] Jiang GJ. Zhuang HR. Li WL. J. Mater. Sci. 2003;38:3559.

[17] Guoquin X. Quncheng F. Meizhuan G, Zhihao J. Mater. Sci. Eng., A 2006:425:318. 
[18] Gras C, Bernsten N, Bernard F, Gaffet E. Intermetallics 2002;10:271

[19] Sharafutdinov MR. Korchagin MA, Shkodich NF, Tolochko BP, Tsygankov PA. Yagubova IY. Nucl. Instrum. Methods Phys. Res. Sect. A 2007;575:149.

[20] Kisi EH, Riley DP, Curfs C. Physica B 2006;385-386:487.

[21] Riley DP, Kisi EH, Phelan D. J. Am. Ceram. Soc. 2006:26:1051.

[22] Riley DP. Kisi EH. Hansen TC. Hewat AW. J. Am. Ceram. Soc. 2002:85:2417.

[23] Riley DP, Kisi EH, Hansen TC. J. Am. Ceram. Soc. 2008:91:3207.

[24] Varma A. Lebrat JP. Chem. Eng. Sci. 1992:47:2179.

[25] Curfs C, Turrillas X. Vaughan GBM. Terry AE, Kvick A. Rodríguez MA. Intermetallics 2007:15:1163.

[26] Mas-Guindal MJ. Turrillas X, Hansen TC, Rodriguez MA. J. Eur. Ceram. Soc. 2008;28:2975.

[27] Hansen TC. Henry PF, Fischer HE. Torregrossa J. Convert P. Meas. Sci. Technol, 2008:19:034001.

[28] ITT Visual Information Solutions, USA. Progran NOeSYS, Version 1.2. 1998.
[29] ITT Visual Information Solutions, USA. Program IDL. Version 5.2, 1998.

[30] OriginLab Corporation, Northampton, MA, 01060. USA. Program Origin Version 8.0, 2007.

[31] Rodríguez-Carvajal J. Physica B 1993;192:55.

[32] Accelrys Software Inc. USA. Program materials studio 4.3, 2008.

[33] Ontotec. HSC Chemistry 6.1, 2006.

[34] Media Cybernetics Inc.. USA. Image-Pro Plus 4.5, 2001.

[35] Nash P. Singleton MF, Murray JL. In: Nash $P$ (Ed.), Phase diagrams of binary nickel alloys. Materials Park $(\mathrm{OH})$ : The Materials Information Society; 1991. p. 3.

[36] Bowen CR, Derby B. Br. Ceram. Trans. 1997:96:25.

[37] Wang Y, Liu ZK, Chen LQ. Acta Mater. 2004;52:2665,

[38] Chung DLL, DeHaven PW, Annold H. Ghosh D. X-ray diffiaction at elevated temperatures, a method for in-situ process analysis. New York: VCH Publishers; 1992. p. 237.

[39] Brown WE, Dollimore D, Galwey AK. In: Banford ECH. Tipper CFH, editors. Chenical kinetics. Reactions in the solid state, vol. 22. Amsterdam: Elsevier; 1980. p. 89. 